Int. J. Electrochem. Sci., 11 (2016) 10795 - 10805

International Journal of

ELECTROCHEMICAL

SCIENCE

www.electrochemsci.org

\title{
Calendula officinalis-mediated biosynthesis of Silver Nanoparticles and their Electrochemical and Optical Characterization
}

\author{
Maged El-Kemary ${ }^{1}$, Eslam Ibrahim ${ }^{2}$, Mohammad, F. A-Ajmi ${ }^{3}$, Shaden A.M. Khalifa ${ }^{4,5}$, \\ Alanazi, A. D ${ }^{6}$., Hesham R. El-Seedi, ${ }^{2,7,8, *}$
}

${ }^{1}$ Division of Photo- and Nanochemistry, Chemistry Department, Faculty of Science, Kafrelsheikh University, 33516 Kafr ElSheikh, Egypt.

${ }^{2}$ Department of Chemistry, Faculty of Science, El-Menoufia University, 32512 Shebin El-Kom, Egypt.

${ }^{3}$ Department of Pharmacognosy, College of Pharmacy, King Saud University, Riyadh 11451, P.O. Box 2457, Saudi Arabia.

${ }^{4}$ Department of Experimental Hematology, Karolinska University Hospital, SE-141 86 Stockholm, Sweden.

${ }^{5}$ Department of Molecular Biosciences, Stockholm University, the Wenner-Gren Institute, SE-106 91 Stockholm, Sweden.

${ }^{6}$ College of Applied Medical Sciences, Shaqra University, KSA, Aldawadmi 11911, P.O.Box 1678, Saudi Arabia.

${ }^{7}$ Division of Pharmacognosy, Department of Medicinal Chemistry, Uppsala University, Box 574, SE75 123, Uppsala, Sweden.

${ }^{8}$ Department of Chemistry, Faculty of Science, University of Malaya, 50603 Kuala Lumpur, Malaysia.

*E-mail: hesham.el-seedi@ fkog.uu.se

doi: $10.20964 / 2016.12 .88$

Received: 24 January 2016 / Accepted: 21 October 2016 / Published: 10 November 2016

The metal nanoparticles synthesis is highly explored field of nanotechnology. The biological methods seem to be more effective. A simple and elegant method is adopted to prepare Silver nanoparticles (AgNPs) in a single step using Calendula officinalis extract (COE) as reducing and stabilizing agent. The plant extract is mixed with $\mathrm{AgNO}_{3}$ to get biosynthesized AgNPs. The biosynthesized AgNPs were both optically and electrochemically characterized by UV-Vis, Infrared spectroscopy, Transmission Electron Microscopy, Fluorescence spectroscopy, Zeta potential and Cyclic Voltammetry. The results showed Calendula officinalis extract is a useful bioreductant for the synthesis of AgNPs. This study infers that the size of biosynthesized AgNPs ranges from 30 to $50 \mathrm{~nm}$. The surface plasmon resonance peak in the UV-Vis absorption spectra shows maximum absorption at $435 \mathrm{~nm}$. Fluorescence spectra of silver nanoparticles, which show an emission peak at $\sim 468 \mathrm{~nm}$ have also been studied. Zeta potential analysis ensured the biosynthesized AgNPs are highly stable. Using this environmentally friendly method of biological AgNPs production supplies rates of biosynthesis facile in comparison with other chemical and engineered routes. The employment of traditional medicine in biosynthesis protocols can 
potentially open new doors in various human health and well-being implications such as cosmetics, foods and medicine.

Keywords: silver nanoparticles (AgNPs), biosynthesis, Calendula officinalis extract.

\section{$\underline{\text { FULL TEXT }}$}

(C) 2016 The Authors. Published by ESG (www.electrochemsci.org). This article is an open access article distributed under the terms and conditions of the Creative Commons Attribution license (http://creativecommons.org/licenses/by/4.0/). 